\title{
Study on Characteristics of Pulsed Eddy Current Field of Copper Belt Detection
}

\author{
Huimin Chen ${ }^{\mathrm{a}}$ Fengshou Zhang ${ }^{\mathrm{b}}$ \\ Henan University of Science and Technology, Luoyang 471003, China \\ ahuiminchen5@163.com, bfengshouzhang@163.com,
}

Keywords: Pulsed eddy current testing,TREE Fourier transform, Eddy current probe.

\begin{abstract}
In order to effectively represent the mechanism of pulsed eddy current testing, the analytical model of harmonic eddy current field above copper strip and plate is worked out in cylindrical coordinate by applying the theory of reflection and transmission of electromagnetic waves and Truncated Region Eigenfunction Expansion method. By Fourier transform of the unit square-wave pulse, analytical model of the pulsed eddy current field is derived, as well as the impedance model and response model of eddy current probe. Those models are simulated by using Mathematical and Comsol, and the relationship is sum up which eddy current probe's impedance and induced voltage vary with thickness, lift-off height, excitation frequency, thus the correctness of analytical model of pulsed eddy current field is validated.
\end{abstract}

\section{Introduction}

Pulsed eddy current field analytical model are an effective tool to understand the mechanism of pulsed eddy current testing, it is based on the analytical model of harmonic eddy current field [1-2]. In this paper, The problem of the copper eddy current field we study to solve can be classified to the problem of multilayer electric conductor eddy current to solve, and harmonic eddy current field analytical model of multilayer electric conductor was founded early in the last century $60 \mathrm{~s}$ by Dodd and his company, and is widely used, but the harmonic eddy current field model Dodd put forward is an integral model [3-4], so its computation process takes long, and is not easy to be high-precision. Later, Fan Mengbao established multilayer electric conductor harmonic eddy current field series analytical model based on the theory of reflection and transmission by Truncated Region Eigenfunction Expansion method (TREE) and the transmission rules of electromagnetic waves in multilayered homogeneous medium[5-6]. The model of analytical model of pulsed eddy current method can be divided into two inspects: [7-8] inverse Fourier transform and inverse Laplace transform.For inverse Fourier transformation ,due to the highness of requirements of mathematics, simple solving,used widely compared with inverse Laplace transform, but the method of modeling accuracy is affected by the Gibbs' effect.

In pulsed eddy current testing, impedance variation of eddy current probe contains information of electrical conductor thickness, conductivity etc theoretically, therefore its impedance analytical model is important to understand the mechanism of eddy current testing and build the inversion model(15th and 16th). In the actual detection, Pulsed eddy current testing technique often obtain the information of copper belt according to the induction voltage on the eddy current probe signal rather than to the change of impedance of the eddy current probe.

This paper based on multilayer electric conductor series harmonic eddy current field analytical model, on the premise of reducing Gibbs effect using Fourier inverse transform method, establishing the analytical model of pulsed eddy current field and impedance model and response model of impedance of eddy current probe under unit pulsed square wave. Finally in order to verify the correctness of the eddy current probe impedance model and response model, simulate and compare the copper plate eddy current probe impedance model and response model under the unit square wave by the Mathematica and Comsol,get the eddy current coil impedance and induction voltage's change law by the thickness of copper belt, heigh, excitation frequency etc. 


\section{Harmonic eddy current field series analytical model in Copper belt}

As shown in fig. 1, layer1 means copper belt,layer2 the air. By the vector magnetic potential of the regional expression in multilayer electric conductor in paper[9] and [10],we can deduce the regional expression of $\mathrm{A}(\mathrm{r}, \mathrm{z})$ in copper belt, as shown in (1).

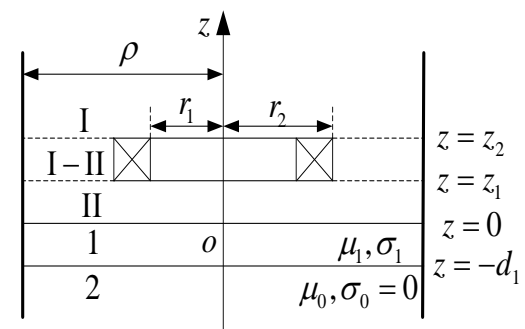

Fig. 1 Geometry model of the eddy current coil on copper belt

$$
\left\{\begin{array}{l}
A_{\mathrm{I}}(r, z)=\sum_{i=1}^{\infty} K\left(C_{\mathrm{I}, i} e^{-\lambda_{0 i} \mathrm{z}}+C_{\mathrm{II}, i} R_{0 i, 1 i}^{\prime} e^{-\lambda_{0 i} \mathrm{z}}\right) \\
A_{\mathrm{I}-\mathrm{II}}(r, z)=\sum_{i=1}^{\infty} K\left(2-e^{\lambda_{0 i}\left(z-z_{2}\right)}-e^{-\lambda_{0 i}\left(z-z_{1}\right)}+R_{0 i, 1 i} e^{-\lambda_{0 i} \mathrm{z}} C_{\mathrm{II}, i}\right) \\
A_{\mathrm{II}}(r, z)=\sum_{i=1}^{\infty} K C_{\mathrm{II}, i}\left(e^{\lambda_{0 i} \mathrm{z}}+R_{0 i, 1 i}^{\prime} e^{-\lambda_{0 i} \mathrm{z}}\right) \\
A_{1}(r, z)=\sum_{i=1}^{\infty} K C_{\mathrm{II}, i}\left(C_{\mathrm{I}, i} e^{\lambda_{0 i} \mathrm{z}}+R_{1 i, 2 i} e^{-\lambda_{0 i} \mathrm{z}}\right) \\
A_{2}(r, z)=\sum_{i=1}^{\infty} K C_{\mathrm{II}, i} C_{2, i} e^{\lambda_{0 i} \mathrm{z}}
\end{array}\right.
$$

Here ,

$$
\begin{aligned}
& K=\mu_{0} J_{c} J_{1}\left(\lambda_{0 i} r\right) \frac{\chi\left(\lambda_{0 i} r_{1}, \lambda_{0 i} r_{2}\right)}{\left[\left(\lambda_{0 i} \rho\right) J_{0}\left(\lambda_{0 i} \rho\right)\right]^{2} \lambda_{0 i}^{2}} \chi\left(\lambda_{0 i} r_{1}, \lambda_{0 i} r_{2}\right)=\int_{\lambda_{0 i} r_{2}}^{\lambda_{0 i} r_{1}} x J_{1}(x) d x, \\
& J_{c}=N_{c} I_{c} /\left[\left(r_{2}-r_{1}\right)\left(z_{2}-z_{1}\right)\right], C_{\mathrm{I}, i}=e^{\lambda_{0 i} z_{2}}-e^{\lambda_{0 i} z_{1}}, C_{\mathrm{II}, i}=e^{-\lambda_{0 i} z_{1}}-e^{-\lambda_{0 i} z_{2}}, \\
& R_{0 i, 1 i}^{\prime}=\frac{\left(\mu_{r 1} \lambda_{0 i}\right)^{2}-\lambda_{1 i}{ }^{2}+e^{-2 \lambda_{1 i} d_{1}}\left[\lambda_{1 i}{ }^{2}-\left(\mu_{r 1} \lambda_{0 i}\right)^{2}\right]}{\left(\lambda_{1 i}+\mu_{r 1} \lambda_{0 i}\right)^{2}-e^{-2 \lambda_{1 i} d_{1}}\left(\lambda_{1 i}-\mu_{r 1} \lambda_{0 i}\right)^{2}}, \\
& R_{1 i, 2 i}=\frac{2 \mu_{r 1} \lambda_{0 i} e^{-2 \lambda_{i i} d_{1}}\left(\lambda_{1 i}-\mu_{r 1} \lambda_{0 i}\right)}{\left(\lambda_{1 i}+\mu_{r 1} \lambda_{0 i}\right)^{2}-e^{-2 \lambda_{i i} d_{1}}\left(\lambda_{1 i}-\mu_{r 1} \lambda_{0 i}\right)^{2}}, \lambda_{1 i}=\sqrt{\lambda_{0 i}{ }^{2}+j \omega \mu_{1} \sigma_{1}},
\end{aligned}
$$

$\sigma_{1}$ - electrical conductivity of copper, $\mu_{1}$ - magnetic permeability of copper belt, $\mu_{\mathrm{r} 1}$ - relative magnetic permeability of copper $d_{1}$ - the thickness of the copper belt, $\sigma 0$ - the electrical conductivity of air, $\mu_{0}$ - magnetic permeability of air, $\mathrm{J}_{c}$ - coil current density, $\mathrm{N}_{\mathrm{c}}-$ coil number of turns, $\mathrm{I}_{\mathrm{c}}$ - exciting current, $\mathrm{r}_{1}$ - inner radius coil , $\mathrm{r} 2$ - outer radius of coil, $\mathrm{z}_{2}, \mathrm{z}_{1}$ - coil on the $\mathrm{z}$ axis, $\rho$-the radius of the solution domain , $\mathrm{J}_{1}(\mathrm{x})$ - the first category 1 order Bessel function , $\lambda_{0 \mathrm{i}}{ }_{1}$ order Bessel function $\mathrm{J}_{1}$ (lambda $0_{\mathrm{I}}$ rho) is the ith root.

\section{The impedance model and response model of the eddy current probe}

\subsection{Impedance model of one coil eddy current probe.}

We can get the induction voltage on the eddy current coil according to the ohm's law $(\mathrm{J}=$ sigma $\mathrm{E})$ is: 


$$
\left\{\begin{array}{l}
V=j \omega 2 \pi n_{c} \int_{r_{1}}^{r_{2}} \int_{z_{1}}^{z_{2}} r A(r, z) d r d z \\
n_{c}=\frac{N_{c}}{\left(r_{2}-r_{1}\right)\left(z_{2}-z_{1}\right)}
\end{array}\right.
$$

In the equation, $\mathrm{n}_{\mathrm{c}}$ - coil turns density.

As shown in Fig.1, with a hollow copper belt placed above the eddy current coil, the induced voltage of the coil is:

$$
\begin{aligned}
& V^{s}=\frac{j 2 \pi \omega \mu_{0} N_{c} I(\omega)}{\left(r_{2}-r_{1}\right)\left(z_{2}-z_{1}\right)} \\
& \int_{r_{1}}^{r_{2}} \int_{z_{1}}^{z_{2}} r \sum_{i=1}^{\infty} \frac{J_{0}\left(\lambda_{0 i} r\right) \chi\left(\lambda_{0 i} r_{1}, \lambda_{0 i} r_{2}\right)}{\left[\left(\lambda_{0 i} \rho\right) J_{0}\left(\lambda_{0 i} \rho\right)\right]^{2} \lambda_{0 i}^{2}}\left[2-e^{\lambda_{0 i}\left(z-z_{2}\right)}-e^{-\lambda_{0 i}\left(z-z_{1}\right)}\right] d r d z \\
& V^{e c}=\frac{j 2 \pi \omega \mu_{0} N_{c} I(\omega)}{\left(r_{2}-r_{1}\right)\left(z_{2}-z_{1}\right)} \\
& \int_{r_{1}}^{r_{2}} \int_{z_{1}}^{z_{2}} r \sum_{i=1}^{\infty} \frac{J_{1}\left(\lambda_{0 i} r\right) \chi\left(\lambda_{0 i} r_{1}, \lambda_{0 i} r_{2}\right)}{\left[\left(\lambda_{0 i} \rho\right) J_{0}\left(\lambda_{0 i} \rho\right)\right]^{2} \lambda_{0 i}^{2}} R_{0 i, 1 i}^{\prime} e^{\lambda_{0 i} z}\left(e^{\lambda_{0 i} z_{2}}-e^{\lambda_{0 i} z_{1}}\right) d r d z
\end{aligned}
$$

In the equation, $\mathrm{Vs}$ - induced voltage in coil excitation magnetic field , Vec - induced voltage in vortex field copper belt,I( $\omega)$-harmonic current.

According to ohm's law: $\mathrm{V}=\mathrm{Z} \times \mathrm{I}$, we can draw the impedance Zair of eddy current coil in the air and the impedance change $\triangle \mathrm{Z}$ caused by the eddy current field:

$$
\begin{aligned}
& Z_{\text {air }}=j 4 \pi \omega \mu_{0} n_{c}^{2} \sum_{i=1}^{\infty} \frac{\chi^{2}\left(\lambda_{0 i} r_{1}, \lambda_{0 i} r_{2}\right)}{\lambda_{0 i}^{5}\left[\rho J_{0}\left(\lambda_{0 i} \rho\right)\right]^{2}}\left[\lambda_{0 i}\left(z_{2}-z_{1}\right)+e^{-\lambda_{0 i}\left(z_{2}-z_{1}\right)}-1\right] \\
& \Delta Z=j 4 \pi \omega \mu_{0} n_{c}^{2} \sum_{i=1}^{\infty} \frac{\chi^{2}\left(\lambda_{0 i} r_{1}, \lambda_{0 i} r_{2}\right)}{\lambda_{0 i}^{5}\left[\lambda_{0 i} \rho J_{0}\left(\lambda_{0 i} \rho\right)\right]^{2}}\left(e^{-\lambda_{0 i} z_{1}}-e^{-\lambda_{0 i} z_{2}}\right)^{2} R_{0 i, 1 i}^{\prime}
\end{aligned}
$$

The eddy current coil impedance $\mathrm{Z}$ on the copper belt:

$$
\text { Z=Zair+ } \quad \triangle Z
$$

\subsection{Induced voltage model of double coil eddy current probe}

According to ohm's law

$$
\left\{\begin{array}{l}
V=j \omega 2 \pi n_{1 c} \int_{r_{3}}^{r_{4}} \int_{z_{3}}^{z_{4}} r A(r, z) d r d z \\
n_{1 c}=\frac{N_{1 c}}{\left(r_{2}-r_{1}\right)\left(z_{2}-z_{1}\right)}
\end{array}\right.
$$

In the equation, $n_{1 c}$-pick up coil turns density, $N_{1 c}$-pick up coil number of turns, $\mathrm{r}_{3}-$ inner radius of pick up coil, $r_{4}$-outer radius of pick up coil, $z_{3}, z_{4}$-coordinates of pick up coil on the $\mathrm{z}$ axis.

According to equation (8), we can conclude induced voltage expressions in different position of pick up coil relative to the excitation coil. The location of the pick up coil relative to the excitation coil is shown in Fig. 2.

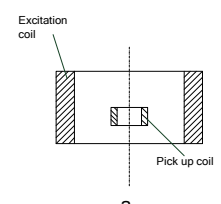

a

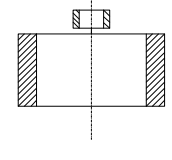

b

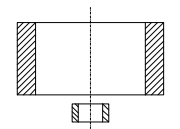

c

Fig. 2 Pick up coil and excitation coil's relative positions 


$$
\left\{\begin{aligned}
V_{a}^{s}= & \frac{j \omega 2 \pi N_{1 c} \mu_{0} I(\omega) N_{c}}{\left(r_{4}-r_{3}\right)\left(z_{4}-z_{3}\right)\left(r_{2}-r_{1}\right)\left(z_{2}-z_{1}\right)} \sum_{i=1}^{\infty} \frac{\chi\left(\lambda_{0 i} r_{1}, \lambda_{0 i} r_{2}\right) \chi\left(\lambda_{0 i} r_{3}, \lambda_{0 i} r_{4}\right)}{\left[\left(\lambda_{0 i} \rho\right) J_{0}\left(\lambda_{0 i} \rho\right)\right]^{2} \lambda_{0 i}^{5}} \\
& \times\left\{2 \lambda_{0 i}\left(z_{4}-z_{1}\right)+\left[e^{\lambda_{0 i}\left(z_{3}-z_{2}\right)}-e^{\lambda_{0 i}\left(z_{4}-z_{2}\right)}-e^{\lambda_{0 i}\left(z_{3}-z_{1}\right)}+e^{\lambda_{0 i}\left(z_{1}-z_{4}\right)}\right]\right\} \\
V_{a}^{e c}= & \frac{j \omega 2 \pi N_{c 1} \mu_{0} I(\omega) N_{c}}{\left(r_{4}-z_{3}\right)\left(z_{4}-z_{3}\right)\left(r_{2}-r_{1}\right)\left(z_{2}-z_{1}\right)} \\
& \sum_{i=1}^{\infty} \frac{\chi\left(\lambda_{0 i} r_{1}, \lambda_{0 i} r_{2}\right) \chi\left(\lambda_{0 i} r_{3}, \lambda_{0 i} r_{4}\right)}{\left[\left(\lambda_{0 i} \rho\right) J_{0}\left(\lambda_{0 i} \rho\right)\right]^{2} \lambda_{0 i}^{5}}\left(e^{-\lambda_{0 i} z_{2}}-e^{-\lambda_{0 i} z_{1}}\right)\left(e^{-\lambda_{0 i} z_{4}}-e^{-\lambda_{0 i} z_{3}}\right) R_{0 i, 1 i}^{\prime}
\end{aligned}\right.
$$

$$
\begin{aligned}
& V_{b}^{s}=\frac{j \omega 2 \pi N_{1 c} \mu_{0} I(\omega) N_{c}}{\left(r_{4}-r_{3}\right)\left(z_{4}-z_{3}\right)\left(r_{2}-r_{1}\right)\left(z_{2}-z_{1}\right)} \sum_{i=1}^{\infty} \frac{\chi\left(\lambda_{01} r_{1}, \lambda_{0 i} r_{2}\right) \chi\left(\lambda_{0 i} r_{3}, \lambda_{0 i} r_{4}\right)}{\left[\left(\lambda_{0 i} \rho\right) J_{0}\left(\lambda_{0 i} \rho\right)\right]^{2} \lambda_{0 i}^{5}} \\
& \times\left(e^{\lambda_{0 i} z_{2}}-e^{\lambda_{0 i} z_{1}}\right)\left(e^{-\lambda_{0 i} z_{3}}-e^{-\lambda_{0 i} z_{4}}\right) \\
& V_{b}^{e c}=\frac{j \omega 2 \pi N_{c 1} \mu_{0} I(\omega) N_{c}}{\left(r_{4}-z_{3}\right)\left(z_{4}-z_{3}\right)\left(r_{2}-r_{1}\right)\left(z_{2}-z_{1}\right)} \\
& \times \sum_{i=1}^{\infty} \frac{\chi\left(\lambda_{0 i} r_{1}, \lambda_{0 i} r_{2}\right) \chi\left(\lambda_{0 i} r_{3}, \lambda_{0 i} r_{4}\right)}{\left[\left(\lambda_{0 i} \rho\right) J_{0}\left(\lambda_{0 i} \rho\right)\right]^{2} \lambda_{0 i}^{5}} e^{-2 \lambda_{0 i} i_{1}}\left(e^{-\lambda_{0 i} z_{2}}-e^{-\lambda_{0 i} z_{1}}\right)\left(e^{-\lambda_{0 i} z_{4}}-e^{-\lambda_{0 i} z_{3}}\right) R_{0 i, 1 i}^{\prime}
\end{aligned}
$$

\section{Pulsed eddy current model based on Fourier transform}

By the Fourier transform,square wave pulse whose cycle is $\mathrm{T}$ can be expanded into a series of Fourier harmonic, as equation(12).

$$
u_{i}(t)=\frac{\tau}{T}+\sum_{i=1}^{N}\left[a_{n} \cos (n \omega t)+b_{n} \sin (n \omega t)\right]
$$

In the equation, $\tau-$ pulse square-wave pulse rate, $T$ - pulse square-wave's cycle, $\omega-$ angular frequency, $\tan =1 / n \pi \sin (n \omega \tau), \quad b n=1 / n \pi[1-\cos (n \omega \tau)], N-$ the total number of Fourier series summation items, $\mathrm{n}$ - Fourier harmonic order.

As a result of Gibbs phenomenon,shock will generate at the edge of the pulse, this will seriously affect the calculation precision of pulsed eddy current model, therefore,introduce the factor $\beta \mathrm{n}$

$$
u_{i}(t)=\frac{\tau}{T}+\beta_{n} \sum_{i=1}^{N}\left[a_{n} \cos (n \omega t)+b_{n} \sin (n \omega t)\right]
$$


In the equation, $\beta \mathrm{n}=\sin (\mathrm{n} \pi / \mathrm{N}) /(\mathrm{n} \pi / \mathrm{N})$.conclude equation (12)and (13)by Mathematica, as shown in Fig. 3 and 4 . We can conclude in the figure that the Fourier series expression is more close to the ideal unit pulse square wave after introducing the coefficient of $\beta \mathrm{n}$.

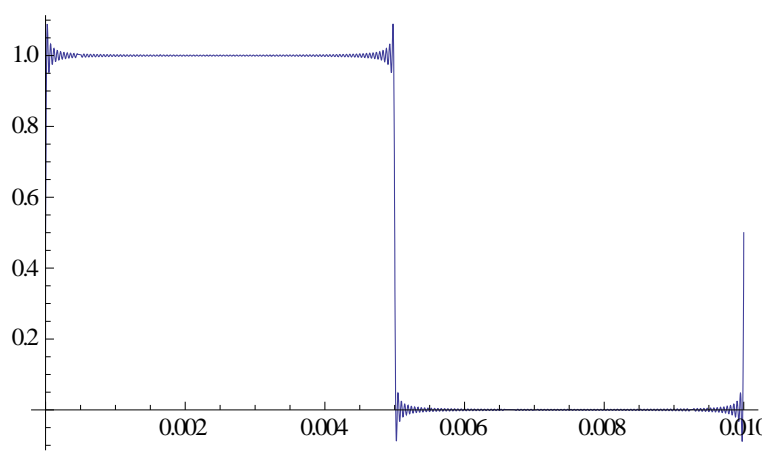

Fig.3 Unit pulse square wave under Fourier series expansion

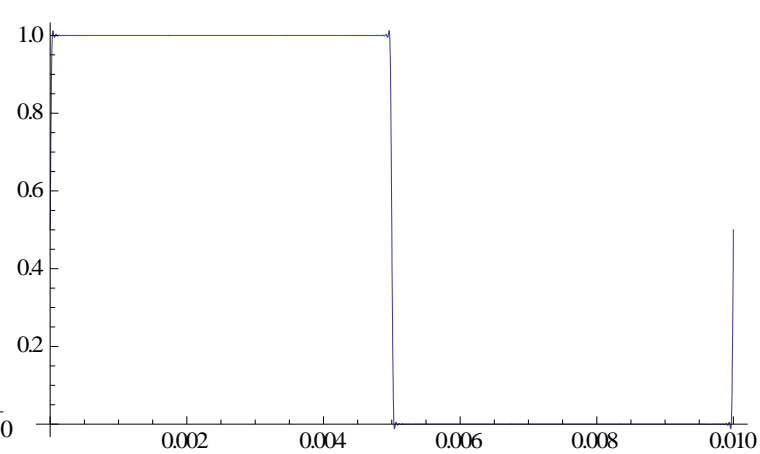

Fig.4 unit pulse square wave after introducing $\beta$ n

Front We have derived impedance model and response model of eddy current probe under the harmonic excitation ,the response of the eddy current probe under unit impulse square wave excitation is corresponding to the sum of the eddy current probe response's linear superposition under a series of harmonic excitation.

\section{Eddy current probe simulation and experiment under unit impulse square wave}

Most commonly double coil eddy current probe use the structure that pick up coil is located in the inner of exciting coil, as shown in Fig.5.

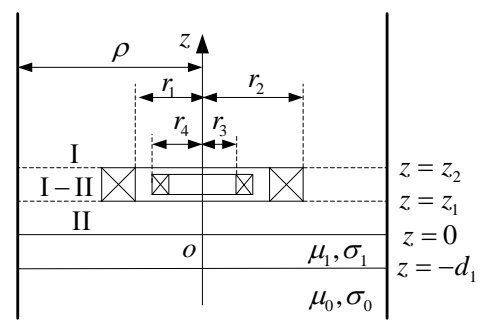

Fig. 5 Drive coil of pick up eddy current inside the probe coil

Eddy current probe parameters are shown in Table 3.

Table 3 Excitation coil and the pickup coil parameters

\begin{tabular}{cll}
\hline Sensor & Parameter & Value(mm $)$ \\
\hline Excitation coil & Inner diameter $/ \mathrm{r} 1$ & 18.5 \\
& Outer diameter $/ \mathrm{r} 2$ & 20.75 \\
& Lift off height/z1 & 0.5 \\
& Coil height/ z2-z1 & 20 \\
& The number of turns & 720 \\
\multirow{3}{*}{ Pick up coil } & Inner diameter /r3 & 8 \\
& Iuter diameter /r4 & 12.5 \\
& Lift off height/z3 & 0.5 \\
& Coil height/z4-z3 & 3 \\
& The number of turns & 216 \\
\hline
\end{tabular}

The change relationship of eddy current probe's induction voltage with the thickness of copper belt, the lift-off height, excitation frequency, are shown in Fig. 6 to 8. 

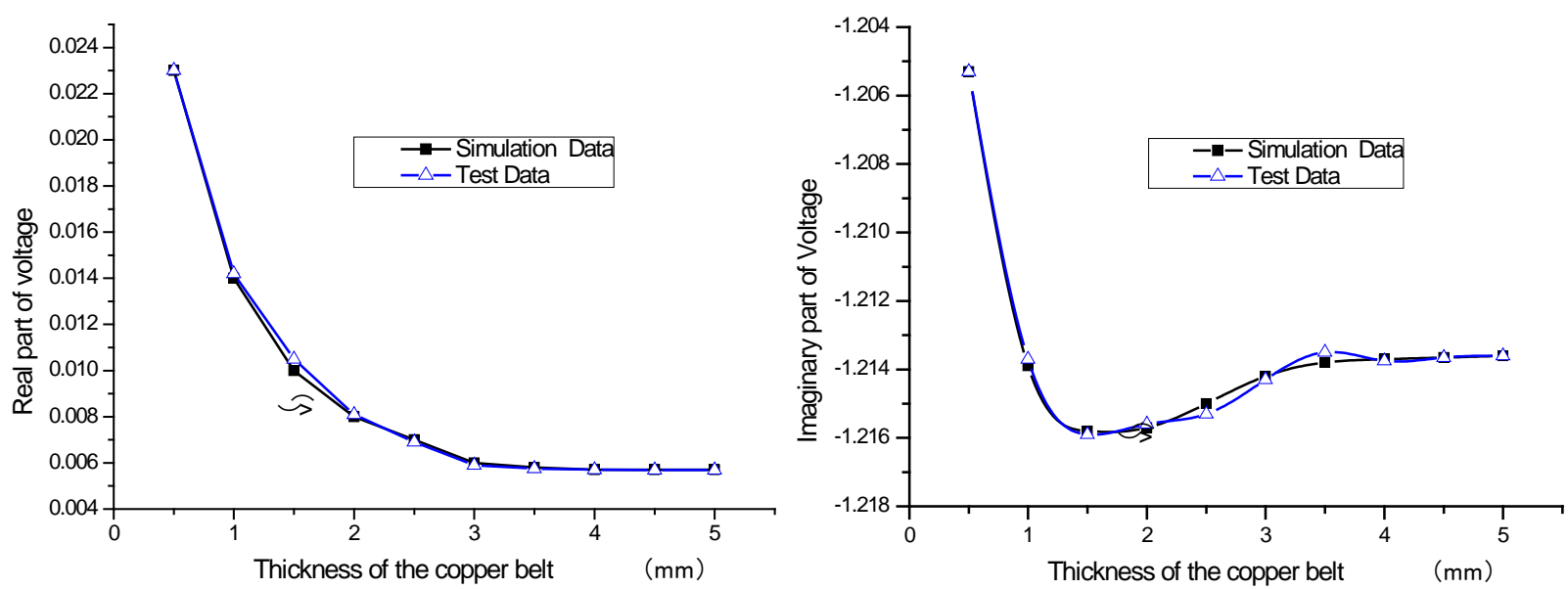

Fig. 6 Pickup coil voltage's change with copper plate thickness
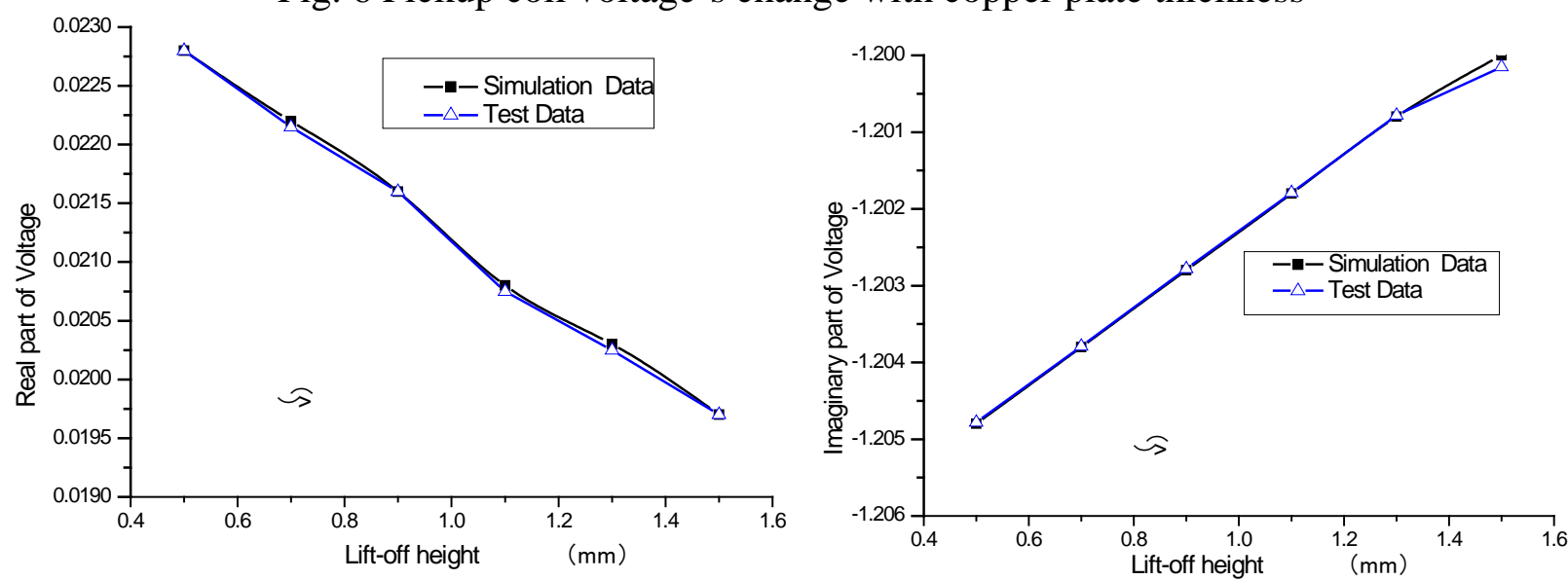

Fig. 7 Pick up coil voltage's change with lift-off height
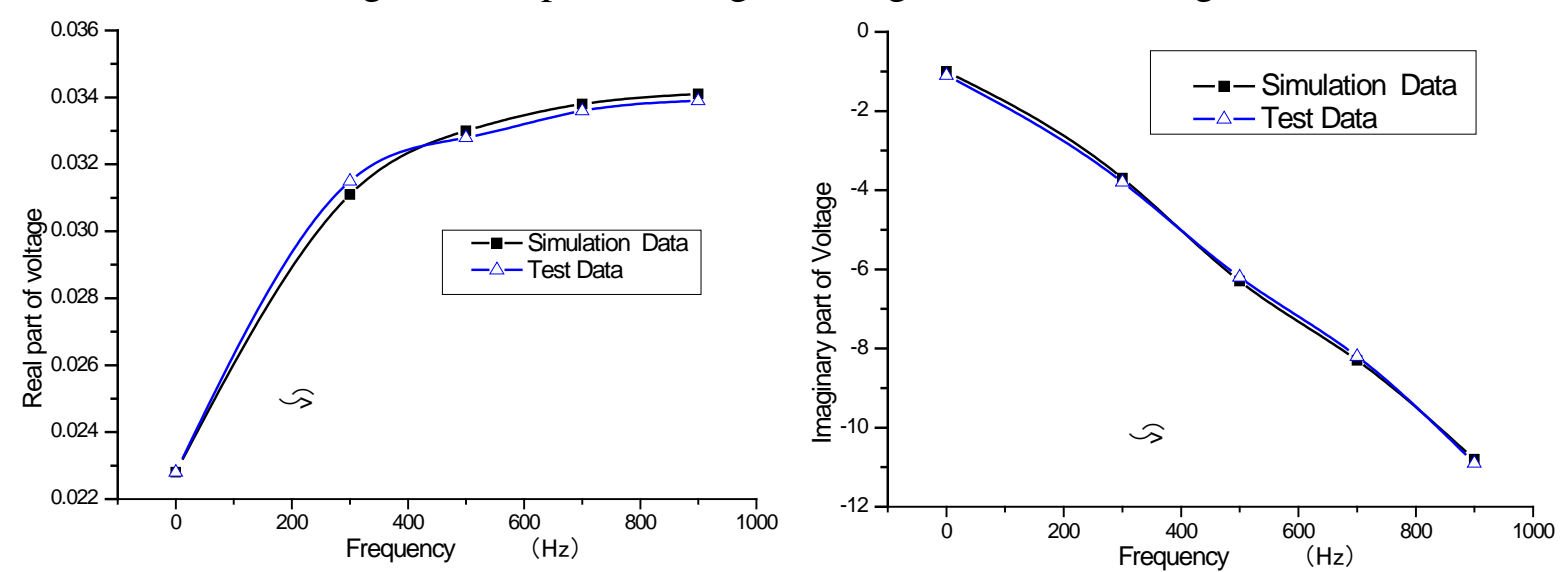

Fig.8 Pick up coil voltage's change with frequency

Fig. 6-8 show that the simulation curve are mainly coincidence with a slight difference by the test and simulation, this prove the impedance and response model of current probe under units pulsed square wave are correct, and the change law of eddy current coil impedance and induced voltage with the excitation frequency, the thickness of the copper belt and lift-off height. By analyzing we can get the main reason of the slight difference in two kinds of simulation results:

(1) In the simulation of model, first of all, $\beta n$ introduced can only reduce but cannot completely eliminate the Gibbs phenomenon, and in the process of simulation using limited number of Fourier harmonic instead of ideal unit pulse square wave, these reasons will make the result of the simulation don't poor.

(2) In finite element simulation, although there is no finite Fourier harmonic's use to instead the ideal unit pulse square wave, and no effect by the influence of Gibbs phenomenon, but the impact of 
grid size's selection in the process of simulation is bigger, the smaller of the grid, the higher precision of the simulation result.

\section{Conclusion}

This paper establishes the copper belt harmonic eddy current analytical model by Phase region characteristic function expansion method and the theory of reflection and transmission of electromagnetic wave, and get the analytical model of pulsed eddy current by Fourier transform, and the eddy current probe's pulse impedance and pick up sensor response expressions under unit square wave. Finally get the eddy current probe impedance and induced voltage change curve under simulation with the excitation frequency, copper thickness and the lift-off height by Mathematic and Comsol, and verify the correctness of the model.

\section{References}

[1]. H. A. Sabbagh, J. C. Treece, RK Murphy. Computer modeling of eddy current nondestructive testing[J]. Mater. Eval. Vol.51(1993) No.11,p.1252-1257.

[2]. C. Reboud, D. Premel, D. Lesselier, B. Bisiaux. Recent advances in simulation of eddy current testing of tubes and experimental validations[J]. Review of Progress in QNDE. Vol. 26(2007),p. 241-248.

[3]. C. D. Dodd, W. E. Deeds. Analytical solutions to eddy-current probe coil problems[J]. J. Appl. Phys. Vol. 39(1968),No. 6,p. 2829-2838.

[4]. THEODOROS T, EPAMEINONDAS K. Series expansions in eddy current nondestructive evaluation models[J]. Journal of Material Processing Technology.Vol.161(2005) No.1-2,p. 343-347.

[5]. Y. Danon, C. Q. Lee, C. Mulligan et a1. Characterizing tantalum sputtered coatings on steel by using eddy currents[J]. IEEE Trans. Magn. Vol. 40(2004), No.4,p. 1826-1832.

[6]. C. Lee, Y. Danon, C. Mulligan. Characterization of niobium, tantalum and chromium sputtered coatings on steel using eddy currents[J]. Surface \& Coating Technology, Vol, 200(2005), No.4,p. 2547-2556.

[7]. T. Kawata, H. Yamada, K. Tsukada. Fourier-transformed eddy current technique to visualize cross-sections of conductive materials[J]. NDT\&E Int. Vol.40(2007) No.7,p.363-367.

[8]. Y. Li, G. Y. Tian, A. Simm. Fast analytical modeling for pulsed eddy current evaluation[J]. NDT\&E Int. Vol.41( 2008) No.6,p. 477-483. 